Proceedings of the Edinburgh Mathematical Society (2003) 46, 147-157 (C)

DOI:10.1017/S0013091502000299 Printed in the United Kingdom

\title{
PRODUCTS OF PAIRWISE TOTALLY PERMUTABLE GROUPS
}

\author{
P. HAUCK ${ }^{1}$, A. MARTÍNEZ-PASTOR ${ }^{2}$ AND M. D. PÉREZ-RAMOS ${ }^{3}$ \\ ${ }^{1}$ Institut für Informatik, Universität Tübingen, Sand 14, 72076 Tübingen, \\ Germany (hauck@informatik.uni-tuebingen.de) \\ ${ }^{2}$ Escuela Universitaria de Informática, Departamento de Matemática Aplicada, \\ Universidad Politécnica de Valencia, Camino de Vera, $s / n$, \\ 46071 Valencia, Spain (anamarti@mat.upv.es) \\ ${ }^{3}$ Departament d'Àlgebra, Universitat de València, C/ Doctor Moliner 50, \\ 46100 Burjassot (València), Spain (Dolores.Perez@uv.es)
}

(Received 4 March 2001)

\begin{abstract}
In this paper finite groups factorized as products of pairwise totally permutable subgroups are studied in the framework of Fitting classes.

Keywords: finite groups; products of groups; Fitting classes
\end{abstract}

AMS 2000 Mathematics subject classification: Primary 20D10; 20D40

\section{Introduction}

All groups considered in this paper are finite. Within the framework of factorized groups, products of totally permutable groups have been widely investigated (cf. $[\mathbf{4}, \mathbf{5}, \mathbf{7}, \mathbf{9}])$. We recall that the subgroups $H$ and $K$ of a group $G$ are totally permutable if every subgroup of $H$ permutes with every subgroup of $K$. Moreover, a group $G$ is the totally permutable product of the subgroups $H$ and $K$ if $G=H K$ and $H$ and $K$ are totally permutable. One of the leading questions in this context asks about properties of the factors which are inherited by the whole group (and vice versa). This can be stated in the following way: assume that $\mathcal{L}$ is a class of groups and $G=H K$ is the product of the totally permutable subgroups $H$ and $K$. We then have the following questions.

(1) Do $H, K \in \mathcal{L}$ imply $G \in \mathcal{L}$ ?

(2) Does $G \in \mathcal{L}$ imply $H, K \in \mathcal{L}$ ?

These questions were answered positively for suitable formations $\mathcal{L}$ containing the formation $\mathcal{U}$ of all supersoluble groups. Moreover, the corresponding natural extensions for products of finitely many pairwise totally permutable groups also hold. We refer to $[\mathbf{1}-\mathbf{3}]$ for details. For the dual type of classes, namely for Fitting classes containing $\mathcal{U}$, the questions mentioned above were considered in [9]. Although they remain open for an 
arbitrary Fitting class $\mathcal{L}$ containing $\mathcal{U}$, positive results were obtained for important types of such Fitting classes, among them Fischer classes. In this paper we take this study further by investigating the case of finitely many factors in the context of Fitting classes. It turns out that whenever $\mathcal{L}$ is a Fitting class containing $\mathcal{U}$ and satisfying either (1) or (2), then the respective extensions for products of finitely many pairwise totally permutable groups hold.

We refer to $[\mathbf{8}]$ for the notation and basic results on classes of groups.

\section{Preliminaries}

In the next lemma we recall a fundamental property of totally permutable groups which will be used often below.

Lemma 2.1 (see Theorem 1 in [5]). Assume that $H$ and $K$ are totally permutable groups. Then $H$ centralizes $K^{\mathcal{N}}$ and $K$ centralizes $H^{\mathcal{N}}$, where $\mathcal{N}$ denotes the class of all nilpotent groups. In particular, $H^{\mathcal{N}}$ and $K^{\mathcal{N}}$ are both normal subgroups of the product $H K$.

The following lemma is an extension of [ $\mathbf{5}$, Corollary 2] for products of pairwise totally permutable groups.

Lemma 2.2. Let $G=G_{1} G_{2} \cdots G_{r}$ be a group such that $G_{1}, G_{2}, \ldots, G_{r}$ are pairwise totally permutable subgroups of $G$. Then $\left[\prod_{i \in I} G_{i}, \prod_{j \in J} G_{j}\right]$ is a nilpotent normal subgroup of $G$, for any $I, J \subseteq\{1,2, \ldots, r\}$ such that $\{I, J\}$ is a partition of $\{1,2, \ldots, r\}$.

Proof. We denote by $T_{i}$ an $\mathcal{N}$-projector of $G_{i}$, for each $i \in\{1,2, \ldots, r\}$. Then $G_{i}=G_{i}^{\mathcal{N}} T_{i}$, for each $i \in\{1,2, \ldots, r\}$. Since the group $G_{j}$ centralizes $G_{i}^{\mathcal{N}}$, for each $i, j \in\{1,2, \ldots, r\}, i \neq j$, by Lemma 2.1 , we have that

$$
\left[\prod_{i \in I} G_{i}, \prod_{j \in J} G_{j}\right]=\left[\prod_{i \in I} G_{i}^{\mathcal{N}} T_{i}, \prod_{j \in J} G_{j}^{\mathcal{N}} T_{j}\right]=\left[\prod_{i \in I} T_{i}, \prod_{j \in J} T_{j}\right] \leqslant\left(\prod_{i \in I} T_{i} \prod_{j \in J} T_{j}\right)^{\prime} .
$$

We notice that $\prod_{i=1}^{r} T_{i}$ is a product of pairwise totally permutable nilpotent subgroups. Then it is a supersoluble group by [6, Theorem 1], and so the result is clear.

Lemma 2.3. Let $T=\langle x\rangle\langle y\rangle$ be a product of two permutable cyclic q-groups, with $q$ an odd prime. Assume that there exists a $q^{\prime}$-group $H$ acting on $T$ by automorphisms such that $T=[H, T]$ and $\langle x\rangle$ and $\langle y\rangle$ are $H$-invariant groups. Then $T$ is an abelian group.

Proof. According to [10, III Satz 11.5], $T$ is metacyclic, that is, there exists a normal subgroup $A$ of $T$ such that $A$ and $T / A$ are cyclic. We now deduce that $T$ is an $M$-group, that is, a group with modular subgroup lattice, by [11, Lemma 2.3.4]. Moreover, since $q$ is odd, $T$ does not involve $Q_{8}$, the quaternion group of order 8 , and so $T$ is an $M^{*}$-group, according to $[\mathbf{1 1}$, p. 58].

Assume that $T$ is non-abelian. Since $T$ is an $M^{*}$-group, by [11, Theorem 2.3.23] there exist characteristic subgroups $R$ and $S$ of $T$ such that $\Phi(T) \leqslant S<R$ and $[R, \operatorname{Aut}(T)] \leqslant$ $S$. Since $T=[H, T]$, with $H \leqslant \operatorname{Aut}(T)$, it is clear that $R<T$. On the other hand, 
$T / \Phi(T) \cong Z_{q} \times Z_{q}$ and so $|T: R|=q$ and $S=\Phi(T)$. Consequently, $[R, H] \leqslant \Phi(T)$. Moreover, $R=[R, H] C_{R}(H)$ by coprime action and we know, by [11, Lemma 2.3.2], that any two of the subgroups of $T$ permute. We may assume that $\langle x\rangle \nsubseteq R$. Then

$$
T=R\langle x\rangle=\Phi(T) C_{R}(H)\langle x\rangle=C_{R}(H)\langle x\rangle .
$$

So

$$
T=[H, T]=\left[H, C_{R}(H)\langle x\rangle\right]=[H,\langle x\rangle] \leqslant\langle x\rangle,
$$

a contradiction which proves the result.

\section{The results}

Theorem 3.1. Let $\mathcal{F}$ be a Fitting class containing $\mathcal{U}$ and satisfying the following property.

(*) If a group $G=H K$ is the product of the totally permutable subgroups $H$ and $K$ such that $H \in \mathcal{F}$ and $K \in \mathcal{F}$, then $G \in \mathcal{F}$.

Let the group $G=G_{1} G_{2} \cdots G_{r}$ be a product of the pairwise totally permutable subgroups $G_{1}, G_{2}, \ldots, G_{r}$. If $G_{i} \in \mathcal{F}$, for all $i \in\{1,2, \ldots, r\}$, then $G \in \mathcal{F}$.

Proof. Assume that the result is false and let $G=G_{1} G_{2} \cdots G_{r}$ be a counterexample where $G_{1}, G_{2}, \ldots, G_{r}$ are pairwise totally permutable $\mathcal{F}$-subgroups of $G$ with $|G|+\left|G_{1}\right|+$ $\cdots+\left|G_{r}\right|$ minimal. We split the proof into the following steps.

(1) We may assume that $G_{2}, \ldots, G_{r}$ are nilpotent groups and that $G_{1}$ is not nilpotent. We denote $H=G_{1}$ and $K=G_{2} \cdots G_{r}$. Moreover, $K \in \mathcal{U}$ and $\left[K, H^{\mathcal{N}}\right]=1$.

If $G_{i} \in \mathcal{N}$ for all $i \in\{1,2, \ldots, r\}$, then $G \in \mathcal{U} \subseteq \mathcal{F}$, by [6, Theorem 1], which is a contradiction. Assume now that there exist $i, j \in\{1,2, \ldots, r\}, i \neq j$, such that $G_{i} \notin \mathcal{N}$ and $G_{j} \notin \mathcal{N}$. From Lemma 2.1 it follows that $\left[G_{k}, G_{t}^{\mathcal{N}}\right]=1$, for all $k, t \in\{1,2, \ldots, r\}, k \neq t$. Then $G_{t} \leqslant C_{G}\left(G_{i}^{\mathcal{N}}\right)<G$ for every $t \neq i$, and $G_{t} \leqslant C_{G}\left(G_{j}^{\mathcal{N}}\right)<G$ for every $t \neq j$. Hence

$$
C_{G}\left(G_{i}^{\mathcal{N}}\right)=\left(\prod_{\substack{t=1 \\ t \neq i}}^{r} G_{t}\right)\left(G_{i} \cap C_{G}\left(G_{i}^{\mathcal{N}}\right)\right)
$$

is a product of pairwise totally permutable subgroups in $\mathcal{F}$, as $C_{G_{i}}\left(G_{i}^{\mathcal{N}}\right) \unlhd G_{i} \in \mathcal{F}$. We conclude that $C_{G}\left(G_{i}^{\mathcal{N}}\right) \in \mathcal{F}$, by the choice of $G$. In a similar way, $C_{G}\left(G_{j}^{\mathcal{N}}\right) \in \mathcal{F}$. Then

$$
G=C_{G}\left(G_{i}^{\mathcal{N}}\right) C_{G}\left(G_{j}^{\mathcal{N}}\right) \in \mathrm{N}_{0}(\mathcal{F})=\mathcal{F}
$$

a contradiction.

Consequently, there exists a unique $i \in\{1,2, \ldots, r\}$ such that $G_{i} \notin \mathcal{N}$. Without loss of generality we may suppose $i=1$. Now the conclusion is clear, by [6, Theorem 1] and Lemma 2.1. 
(2) $K^{G} \cap H \in \mathcal{N}$ and $K^{G} \in \mathcal{U}$.

Since $\left[K, H^{\mathcal{N}}\right]=1$ we can deduce that $K^{G} \cap H^{\mathcal{N}} \leqslant Z\left(K^{G} \cap H\right)$ and so $K^{G} \cap H \in$ $\mathcal{N}$. Finally, since $K^{G}=\left(K^{G} \cap H\right) K$ is a product of pairwise totally permutable nilpotent subgroups, then $K^{G} \in \mathcal{U}$, by [6, Theorem 1].

(3) There exists a prime number $p$ such that $G=H^{\mathcal{N}} H_{p} K^{G}$, with $H_{p}$ a Sylow $p$ subgroup of $H$.

Since $H^{\mathcal{N}} H_{q} K^{G}$ is a normal subgroup of $G$, for all primes $q$, where $H_{q}$ is a Sylow $q$-subgroup of $H$, the result follows after taking into account the choice of $G$.

(4) For all primes $q \neq p, H^{\mathcal{N}} H_{q}[H, K]$ is a normal $\mathcal{F}$-subgroup of $G$, where $H_{q}$ is a Sylow $q$-subgroup of $H$.

We notice first that $H^{\mathcal{N}} H_{q}[H, K]$ is a normal subgroup of $G=H K$ contained in $H^{\mathcal{N}} K^{G}$ by (3). Then the result follows from (2) and this fact.

(5) $H^{\mathcal{N}} H_{p}\left[H_{p}, K\right] \notin \mathcal{F}$.

Suppose that $H^{\mathcal{N}} H_{p}\left[H_{p}, K\right] \in \mathcal{F}$. Since $H^{\mathcal{N}} H_{p}\left[H_{p}, K\right]=\left(H^{\mathcal{N}} H_{p}\right)^{G}$ is a normal subgroup of $G$, then

$$
H^{G}=H[H, K]=\left(H^{\mathcal{N}} H_{p}\left[H_{p}, K\right]\right)\left(\prod_{q \neq p} H^{\mathcal{N}} H_{q}[H, K]\right) \in \mathrm{N}_{0}(\mathcal{F})=\mathcal{F},
$$

by (4). Consequently, $G=H^{G} K^{G} \in \mathrm{N}_{0}(\mathcal{F})=\mathcal{F}$, a contradiction which proves Step (5).

(6) $G=H^{\mathcal{N}} H_{p} K$.

If $H^{\mathcal{N}} H_{p} K<G$, then $H^{\mathcal{N}} H_{p} K \in \mathcal{F}$ by the choice of $G$. But this contradicts Step (5), since $H^{\mathcal{N}} H_{p}\left[H_{p}, K\right]$ is a normal subgroup of $H^{\mathcal{N}} H_{p} K$.

(7) $H / H^{\mathcal{N}}$ is a $p$-group.

This follows from (6) by the choice of $\left(G_{1}, \ldots, G_{r}\right)$.

(8) $p \leqslant q$ for all primes $q$ dividing $|K|$ and $G=H^{\mathcal{N}} H_{p} K_{p} K_{p^{\prime}}$, where $K_{p}$ is a Sylow $p$-subgroup of $K$ and $K_{p^{\prime}}$ is a Hall $p^{\prime}$-subgroup of $K$. Moreover, $K_{p^{\prime}}$ is a normal subgroup of $G$.

Suppose that $p \geqslant q$, for all primes $q$ dividing $|K|$. Since $H_{p} K$ is a supersoluble group by [6, Theorem 1], we can deduce that $H=H^{\mathcal{N}} H_{p}$ is a subnormal subgroup of $G=H^{\mathcal{N}} H_{p} K$. Hence, $G=K^{G} H \in \mathrm{N}_{0}(\mathcal{F})=\mathcal{F}$, a contradiction. Consequently, there exists a prime $q$ dividing $|K|$ with $p<q$.

Let

$$
\begin{aligned}
& \pi(K) \cup\{p\}=\left\{p_{1}, p_{2}, \ldots, p_{t}=p, p_{t+1}, \ldots, p_{n}\right\}, \\
& \text { with } p_{1}<p_{2}<\cdots<p_{t}=p<p_{t+1}<\cdots<p_{n} .
\end{aligned}
$$


We let $\pi=\left\{p, p_{t+1}, \ldots, p_{n}\right\}$ and $\pi^{\prime}=(\pi(K) \cup\{p\}) \backslash \pi$. Since $H_{p} K$ is a supersoluble group, $K_{\pi^{\prime}}$ normalizes $H_{p} K_{\pi}$, where $K_{\pi^{\prime}}$ and $K_{\pi}$ are a Hall $\pi^{\prime}$-subgroup and the Hall $\pi$-subgroup of $K$, respectively. Hence $H^{\mathcal{N}} H_{p} K_{\pi}$ is a normal subgroup of $G$. Assume that $H^{\mathcal{N}} H_{p} K_{\pi}<G$. We notice that $K_{\pi}=O_{\pi}\left(G_{2}\right) \cdots O_{\pi}\left(G_{r}\right)$ is a product of pairwise totally permutable nilpotent subgroups each of which is totally permutable with $H$. It then follows that $H^{\mathcal{N}} H_{p} K_{\pi} \in \mathcal{F}$ by the choice of $G$. Therefore, $G=\left(H^{\mathcal{N}} H_{p} K_{\pi}\right) K^{G} \in \mathrm{N}_{0}(\mathcal{F})=\mathcal{F}$, a contradiction which implies that $G=H^{\mathcal{N}} H_{p} K_{\pi}$. Now, by the choice of $\left(G_{1}, \ldots, G_{r}\right)$, it follows that $K=K_{\pi}$ and $p \leqslant q$ for all primes $q \in \pi(K)$. Since $H_{p} K \in \mathcal{U}$, it is clear that $K_{p^{\prime}}$ is a normal subgroup of $G=H^{\mathcal{N}} H_{p} K_{p} K_{p^{\prime}}$ and we are done.

(9) $K$ is a normal $p^{\prime}$-subgroup of $G$.

We notice that $K_{p^{\prime}}=O_{p^{\prime}}\left(G_{2}\right) \cdots O_{p^{\prime}}\left(G_{r}\right)$. If $H K_{p^{\prime}}<G$, then $H K_{p^{\prime}} \in \mathcal{F}$ by the choice of $G$. Now, since $H^{\mathcal{N}} K_{p^{\prime}}$ is a normal subgroup of $G$ and $G / H^{\mathcal{N}} K_{p^{\prime}}$ is a pgroup by (8), it follows that $H K_{p^{\prime}}$ is a subnormal subgroup of $G$. This means that $G=\left(H K_{p^{\prime}}\right) K^{G} \in \mathrm{N}_{0}(\mathcal{F})=\mathcal{F}$, a contradiction. Hence $G=H K_{p^{\prime}}$ and $K=K_{p^{\prime}}$ by the choice of $\left(G_{1}, \ldots, G_{r}\right)$. By (8), $K$ is normal in $G$.

(10) For all $j \in\{2, \ldots, r\}, G_{j}=\left[G_{j}, H\right]$. Moreover, $\prod_{k=1}^{t} G_{j_{k}}=\left[\prod_{k=1}^{t} G_{j_{k}}, H\right]$ for each set of indices $\left\{j_{1}, \ldots, j_{t}\right\} \subseteq\{2, \ldots, r\}$. In particular, $H^{G}=G$ and $K=[H, K]$ is a nilpotent group.

First, we remark that for every $j \in\{2, \ldots, r\},\left[G_{j}, H\right]=\left[G_{j}, H_{p}\right] \leqslant G_{j}$ because $H=H^{\mathcal{N}} H_{p},\left[H^{\mathcal{N}}, K\right]=1$ and $H_{p} K$ is a supersoluble group, with $p$ the smallest prime dividing its order. Now, since $G_{j}$ is a $p^{\prime}$-group, by coprime action we know that $G_{j}=\left[G_{j}, H_{p}\right] C_{G_{j}}\left(H_{p}\right)$, for all $j \in\{2, \ldots, r\}$. Then we have that

$$
H^{G}=\left\langle H^{G_{2} \cdots G_{r}}\right\rangle \leqslant H\left(\prod_{j=2}^{r}\left[G_{j}, H_{p}\right]\right) \leqslant H^{G} .
$$

Since $H^{G}=H\left(\prod_{j=2}^{r}\left[G_{j}, H_{p}\right]\right)$ is a product of pairwise totally permutable subgroups in $\mathcal{F}$, if we assume that $H^{G}<G$, then by the choice of $G$ we deduce that $H^{G} \in \mathcal{F}$ and $G=K H^{G} \in \mathrm{N}_{0}(\mathcal{F})=\mathcal{F}$, a contradiction. Hence

$$
G=H^{G}=H\left(\prod_{j=2}^{r}\left[G_{j}, H_{p}\right]\right) .
$$

By the choice of $\left(G_{1}, \ldots, G_{r}\right)$ we conclude that $G_{j}=\left[G_{j}, H_{p}\right]=\left[G_{j}, H\right]$, for all $j \in\{2, \ldots, r\}$.

Now, if we take $\left\{j_{1}, \ldots, j_{t}\right\} \subseteq\{2, \ldots, r\}$, then we have that

$$
\prod_{k=1}^{t} G_{j_{k}}=\prod_{k=1}^{t}\left[G_{j_{k}}, H\right] \leqslant\left[\prod_{k=1}^{t} G_{j_{k}}, H\right] \leqslant \prod_{k=1}^{t} G_{j_{k}} .
$$

In particular, $K=[H, K]$ is a nilpotent group, by Lemma 2.2 . 
(11) For all $j \in\{2, \ldots, r\}, G_{j}$ is an abelian group and, moreover, $H$ normalizes each subgroup of $G_{j}$.

Choose any $j \in\{2, \ldots, r\}$. We claim that $H$ does not centralize any non-trivial Sylow subgroup of $G_{j}$. Assume that it does and let $\left(G_{j}\right)_{q} \neq 1$, a Sylow $q$-subgroup of $G_{j}$, for some prime $q$, such that $\left[\left(G_{j}\right)_{q}, H\right]=1$. Then $H^{G} \leqslant H\left(\prod_{1 \neq i \neq j} G_{i}\right)\left(G_{j}\right)_{q^{\prime}}$ and, by (10), we deduce that $G=H\left(\prod_{1 \neq i \neq j} G_{i}\right)\left(G_{j}\right)_{q^{\prime}}$. Now, by the choice of $\left(G_{1}, \ldots, G_{r}\right)$, we obtain that $G_{j}=\left(G_{j}\right)_{q^{\prime}}$ and $\left(G_{j}\right)_{q}=1$, a contradiction.

Since $H_{p}\left(G_{j}\right)_{q}$ is a product of totally permutable subgroups and it is a supersoluble group, it follows that $H_{p}$ normalizes each subgroup of $\left(G_{j}\right)_{q}$ but does not centralize $\left(G_{j}\right)_{q}$, for all primes $q \in \pi\left(G_{j}\right)$. By [5, Lemma 1], $\left(G_{j}\right)_{q}$ is an abelian group, for all primes $q \in \pi\left(G_{j}\right)$, and hence $G_{j}$ is an abelian group.

Again, since $H_{p} G_{j}$ is a supersoluble group which is a product of two totally permutable subgroups, and $p$ is the smallest prime dividing its order, we deduce that $H_{p}$ normalizes each subgroup of $G_{j}$. Now, since $\left[H^{\mathcal{N}}, G_{j}\right]=1$, the result follows by (7).

(12) $G_{j}$ is a cyclic $p_{j}$-group, for some prime $p_{j}$, for all $j \in\{2, \ldots, r\}$.

Choose any $j \in\{2, \ldots, r\}$. Since $G_{j}$ is abelian by (11), it is a direct product of cyclic subgroups of prime power orders. Let $G_{j}=\times_{i} T_{j_{i}}, T_{j_{i}} \cong Z_{p_{i}^{\alpha_{i}}}$ for some primes $p_{i}>2$ and some integers $\alpha_{i} \geqslant 0$ for each $i$. Then $G=H\left(\prod_{1 \neq k \neq j} G_{k}\right)\left(\times_{i} T_{j_{i}}\right)$ is a product of pairwise totally permutable subgroups in $\mathcal{F}$. Now,

$$
|H|+\sum_{1 \neq k \neq j}\left|G_{k}\right|+\sum_{i}\left|T_{j_{i}}\right|<\left|G_{1}\right|+\cdots+\left|G_{r}\right|,
$$

since

$$
\sum_{i}\left|T_{j_{i}}\right|<\prod_{i}\left|T_{j_{i}}\right|
$$

unless $G_{j}=T_{j_{i}}$ for some index $j_{i}$. It follows that each $G_{j}$ is a cyclic $p_{j}$-group, for some prime $p_{j}$, by the choice of $\left(G_{1}, \ldots, G_{r}\right)$.

(13) $K=G_{2} \cdots G_{r}$ is an abelian group.

Since $K$ is a nilpotent group by (10), it suffices to show that any Sylow $q$-subgroup of $K$, for any prime $q$, is abelian. Take any pair of indices $i, j \in\{2, \ldots, r\}$ such that $G_{i}$ and $G_{j}$ are $q$-groups and denote $T_{i j}=G_{i} G_{j}$. By $(10), T_{i j}=\left[T_{i j}, H\right]=$ $\left[T_{i j}, H_{p}\right]$. Moreover, by (12), $T_{i j}$ is the product of two permutable cyclic $H$-invariant $q$-subgroups, where $q$ is an odd prime (we recall that $p<q$ ). Then $T_{i j}$ is an abelian group by Lemma 2.3. This means that $\left[G_{i}, G_{j}\right]=1$ for every pair of $q$-groups $G_{i}$ and $G_{j}$. Consequently, we deduce that any Sylow $q$-subgroup of $K$ is abelian, by (12), and the result follows.

(14) The final contradiction.

Since $K=G_{2} \cdots G_{r}$ is an abelian group and $H$ normalizes each subgroup of $G_{r}$, it is clear that $H G_{2} \cdots G_{r-1}$ is totally permutable with $G_{r}$. If $H G_{2} \cdots G_{r-1}$ is a 
proper subgroup of $G$, then it is an $\mathcal{F}$-group by the choice of $G$. Consequently, $G=\left(H G_{2} \cdots G_{r-1}\right) G_{r}$ is a product of two totally permutable subgroups in $\mathcal{F}$. From our assumption we obtain that $G \in \mathcal{F}$, a contradiction. This implies that $G=H G_{2} \cdots G_{r-1}$. By the choice of $\left(G_{1}, \ldots, G_{r}\right)$ we have that $G \in \mathcal{F}$, the final contradiction.

Theorem 3.2. Let $\mathcal{F}$ be a Fitting class containing $\mathcal{U}$ and satisfying the following property.

(**) If a group $G=H K$ is the product of the totally permutable subgroups $H$ and $K$ such that $G \in \mathcal{F}$, then $H \in \mathcal{F}$ and $K \in \mathcal{F}$.

Let the group $G=G_{1} G_{2} \cdots G_{r}$ be a product of the pairwise totally permutable subgroups $G_{1}, G_{2}, \ldots, G_{r}$. If $G \in \mathcal{F}$, then $G_{i} \in \mathcal{F}$ for all $i \in\{1,2, \ldots, r\}$.

Proof. Assume that the result is false and let $G=G_{1} G_{2} \cdots G_{r} \in \mathcal{F}$ be a counterexample where $G_{1}, G_{2}, \ldots, G_{r}$ are pairwise totally permutable subgroups of $G$, not all of them in $\mathcal{F}$, with $|G|+\left|G_{1}\right|+\cdots+\left|G_{r}\right|$ minimal. We split the proof into the following steps.

(1) We may assume that $G_{2}, \ldots, G_{r}$ are nilpotent groups and $G_{1}$ is not nilpotent. We denote $H=G_{1}$ and $K=G_{2} \cdots G_{r}$. Moreover, $K \in \mathcal{U}$ and $\left[K, H^{\mathcal{N}}\right]=1$.

Obviously, not all $G_{1}, G_{2}, \ldots, G_{r}$ are nilpotent. If we assume that there exists $i, j \in\{1,2, \ldots, r\}, i \neq j$, such that $G_{i} \notin \mathcal{N}$ and $G_{j} \notin \mathcal{N}$, we can deduce, as in Theorem 3.1, Step (1), that $C_{G}\left(G_{i}^{\mathcal{N}}\right)=\left(\prod_{t=1, t \neq i}^{r} G_{t}\right)\left(G_{i} \cap C_{G}\left(G_{i}^{\mathcal{N}}\right)\right)$ is a proper normal subgroup of $G$. By the choice of $G$, we obtain that $G_{t} \in \mathcal{F}$, for all $t \neq i$. In a similar way, using $G_{j}$, we deduce that $G_{l} \in \mathcal{F}$, for all $l \neq j$. Then $G_{k} \in \mathcal{F}$ for all $k \in\{1,2, \ldots, r\}$, a contradiction.

Consequently, there exists a unique $i \in\{1,2, \ldots, r\}$ such that $G_{i} \notin \mathcal{N}$. Without loss of generality we may assume that $i=1$. The conclusion is now clear by $[\mathbf{6}$, Theorem 1] and Lemma 2.1.

(2) $K^{G} \cap H \in \mathcal{N}$ and $K^{G} \in \mathcal{U}$.

We can argue as in Theorem 3.1, Step (2).

(3) There exists a prime number $p$ such that $G=H^{\mathcal{N}} H_{p} K^{G}$, with $H_{p}$ a Sylow $p$ subgroup of $H$.

Assume that $H^{\mathcal{N}} H_{q} K^{G}<G$, for all primes $q$, where $H_{q}$ is a Sylow $q$-subgroup of $H$. For every prime $q$, since $H^{\mathcal{N}} H_{q} K^{G}$ is a normal subgroup of $G$, we have that $H^{\mathcal{N}} H_{q} K^{G} \in \mathrm{S}_{n}(\mathcal{F})=\mathcal{F}$. But $H^{\mathcal{N}} H_{q} K^{G}=H^{\mathcal{N}} H_{q}\left(K^{G} \cap H\right) K$ is a product of pairwise totally permutable subgroups. By the choice of $G$ we deduce that $H^{\mathcal{N}} H_{q}\left(K^{G} \cap H\right) \in \mathcal{F}$. In particular, $H^{\mathcal{N}} H_{q} \in \mathrm{S}_{n}(\mathcal{F})=\mathcal{F}$, for all primes $q$, and so $H \in \mathrm{N}_{0}(\mathcal{F})=\mathcal{F}$, a contradiction. 
(4) For all primes $q \neq p, H^{\mathcal{N}} H_{q} \in \mathcal{F}$, where $H_{q}$ is a Sylow $q$-subgroup of $H$. Moreover, $H^{\mathcal{N}} H_{p} \notin \mathcal{F}$.

We notice that $H^{\mathcal{N}} H_{q}$ is contained in $H^{\mathcal{N}}\left(K^{G} \cap H\right)$ by $(3)$. But $H^{\mathcal{N}}\left(K^{G} \cap H\right) \in \mathcal{F}$ because it is the product of two normal $\mathcal{F}$-subgroups of $H$. Then $H^{\mathcal{N}} H_{q} \in \mathrm{s}_{n}(\mathcal{F})=$ $\mathcal{F}$. Finally, if $H^{\mathcal{N}} H_{p} \in \mathcal{F}$, then $H \in \mathrm{N}_{0}(\mathcal{F})=\mathcal{F}$, a contradiction which proves (4).

(5) $H / H^{\mathcal{N}}$ is a $p$-group, $p \leqslant q$ for all primes $q$ dividing $|K|$ and $G=H^{\mathcal{N}} H_{p} K_{p} K_{p^{\prime}}$, where $K_{p}$ is a Sylow $p$-subgroup of $K$ and $K_{p^{\prime}}$ is a Hall $p^{\prime}$-subgroup of $K$. Moreover, $K_{p^{\prime}}$ is a normal subgroup of $G$.

Since $H^{\mathcal{N}} H_{p}\left[H_{p}, K\right]=\left(H^{\mathcal{N}} H_{p}\right)^{G}$ is a normal subgroup of $G$, we have that $H^{\mathcal{N}} H_{p}\left[H_{p}, K\right] \in \mathcal{F}$.

First we claim that there exists a prime $q$ dividing $|K|$ with $p<q$. Otherwise, we can obtain, as in Theorem 3.1, Step (8), that $H^{\mathcal{N}} H_{p}$ is a subnormal subgroup of $H^{\mathcal{N}} H_{p}\left[H_{p}, K\right]$. Then $H^{\mathcal{N}} H_{p} \in \mathrm{s}_{n}(\mathcal{F})=\mathcal{F}$, which contradicts Step $(4)$.

Let

$$
\begin{aligned}
& \pi(K) \cup\{p\}=\left\{p_{1}, p_{2}, \ldots, p_{t}=p, p_{t+1}, \ldots, p_{n}\right\} \\
& \qquad \text { with } p_{1}<p_{2}<\cdots<p_{t}=p<p_{t+1}<\cdots<p_{n} .
\end{aligned}
$$

We let $\pi=\left\{p, p_{t+1}, \ldots, p_{n}\right\}$ and $\pi^{\prime}=(\pi(K) \cup\{p\}) \backslash \pi$. We recall that $K=$ $G_{2} \cdots G_{r}=K_{\pi} K_{\pi^{\prime}}$ is a supersoluble group, where $K_{\pi^{\prime}} \in \operatorname{Hall}_{\pi^{\prime}}(K)$ and $K_{\pi} \in$ $\operatorname{Hall}_{\pi}(K)$. We may assume that $K_{\pi^{\prime}}=O_{\pi^{\prime}}\left(G_{2}\right) \cdots O_{\pi^{\prime}}\left(G_{r}\right)$ is a product of pairwise totally permutable nilpotent subgroups each of which is totally permutable with $H$. Then $H_{p} K_{\pi^{\prime}}$ is a supersoluble group by [6, Theorem 1]. Consequently, $K_{\pi^{\prime}}$ normalizes both $K_{\pi}$ and $H_{p}$ and so we have that

$$
\left[H_{p}, K\right]=\left[H_{p}, K_{\pi^{\prime}}\right]\left[H_{p}, K_{\pi}\right] \leqslant H_{p}\left[H_{p}, K_{\pi}\right] .
$$

Therefore, $H^{\mathcal{N}} H_{p}\left[H_{p}, K_{\pi}\right]=H^{\mathcal{N}} H_{p}\left[H_{p}, K\right]$ is a normal $\mathcal{F}$-subgroup of $G$. On the other hand, arguing as above, we have that $H_{p} K_{\pi}$ is also a supersoluble group. This implies that $K_{\pi}$ is a subnormal subgroup of $H_{p} K_{\pi}$. Hence $H^{\mathcal{N}} H_{p} K_{\pi}=$ $H^{\mathcal{N}} H_{p}\left[H_{p}, K_{\pi}\right] K_{\pi} \in \mathrm{N}_{0}(\mathcal{F})=\mathcal{F}$.

If $H^{\mathcal{N}} H_{p} K_{\pi}<G$, then $H^{\mathcal{N}} H_{p} \in \mathcal{F}$, by the choice of $G$, which contradicts Step (4). So we may assume that $G=H^{\mathcal{N}} H_{p} K_{\pi}$. Now, by the choice of $\left(G_{1}, \ldots, G_{r}\right)$, we can deduce that $H=H^{\mathcal{N}} H_{p}, K=K_{\pi}$ and $p \leqslant q$ for all primes $q \in \pi(K)$. Moreover, since $H_{p} K \in \mathcal{U}$, it is clear that $K_{p^{\prime}}$ is a normal subgroup of $G$, and the result follows.

(6) $K$ is a normal $p^{\prime}$-subgroup of $G$.

Since $H^{\mathcal{N}} K_{p^{\prime}}$ is a normal subgroup of $G$ and $G / H^{\mathcal{N}} K_{p^{\prime}}$ is a $p$-group by (5), then $H K_{p^{\prime}}$ is a subnormal subgroup of $G$. In particular, $H K_{p^{\prime}} \in \mathrm{s}_{n}(\mathcal{F})=\mathcal{F}$. If $H K_{p^{\prime}}<$ $G$, then by the choice of $G$ we can deduce that $H \in \mathcal{F}$, a contradiction. Hence $G=H K_{p^{\prime}}$ and $K=K_{p^{\prime}}$ by the choice of $\left(G_{1}, \ldots, G_{r}\right)$. 
(7) For all $j \in\{2, \ldots, r\}, G_{j}=\left[G_{j}, H\right]$. Moreover,

$$
\prod_{k=1}^{t} G_{j_{k}}=\left[\prod_{k=1}^{t} G_{j_{k}}, H\right]
$$

for each set of indices $\left\{j_{1}, \ldots, j_{t}\right\} \subseteq\{2, \ldots, r\}$. In particular, $H^{G}=G$ and $K=$ $[H, K]$ is a nilpotent group.

From (1), (5) and (6), we can argue as in Theorem 3.1, Step (10), to obtain that $\left[G_{j}, H\right]=\left[G_{j}, H_{p}\right] \leqslant G_{j}$, for every $j \in\{2, \ldots, r\}$, and that

$$
H^{G}=H\left(\prod_{j=2}^{r}\left[G_{j}, H_{p}\right]\right) .
$$

In particular, $H^{G}$ is a normal subgroup of $G \in \mathcal{F}$, which is a product of pairwise totally permutable subgroups. If $H^{G}<G$, then $H \in \mathcal{F}$ by the choice of $G$, a contradiction. Consequently,

$$
G=H^{G}=H\left(\prod_{j=2}^{r}\left[G_{j}, H_{p}\right]\right)
$$

and, by the choice of $\left(G_{1}, \ldots, G_{r}\right)$, we conclude that $G_{j}=\left[G_{j}, H_{p}\right]=\left[G_{j}, H\right]$, for all $j \in\{2, \ldots, r\}$.

The remainder follows easily as in Theorem 3.1, Step (10).

(8) For all $j \in\{2, \ldots, r\}, G_{j}$ is an abelian group and, moreover, $H$ normalizes each subgroup of $G_{j}$.

This follows by arguing as in Theorem 3.1, Step (11).

(9) $G_{j}$ is a cyclic $p_{j}$-group, for some prime $p_{j}$, for all $j \in\{2, \ldots, r\}$.

Arguing as in Theorem 3.1, Step (12), and with the same notation, we obtain that, for any $j \in\{2, \ldots, r\}, G=H\left(\prod_{1 \neq k \neq j} G_{k}\right)\left(\times_{i} T_{j_{i}}\right)$ is a product of pairwise totally permutable subgroups with

$$
|H|+\sum_{1 \neq k \neq j}\left|G_{k}\right|+\sum_{i}\left|T_{j_{i}}\right|<\left|G_{1}\right|+\cdots+\left|G_{r}\right|,
$$

unless $G_{j}$ is a cyclic $p_{j}$-subgroup, for some prime $p_{j}$. Since $H \notin \mathcal{F}$, the result follows analogously by the choice of $\left(G_{1}, \ldots, G_{r}\right)$.

(10) $K=G_{2} \cdots G_{r}$ is an abelian group.

This follows from Lemma 2.3, Steps (7) and (9), arguing as in Theorem 3.1, Step (13). 
(11) The final contradiction.

By Steps (8) and (10), it is clear that $H G_{2} \cdots G_{r-1}$ is totally permutable with $G_{r}$. Then we can apply our assumption on the group $G=\left(H G_{2} \cdots G_{r-1}\right) G_{r} \in \mathcal{F}$ to obtain that $H G_{2} \cdots G_{r-1} \in \mathcal{F}$. By the choice of $\left(G, G_{1}, \ldots, G_{r}\right)$ we can deduce that $H \in \mathcal{F}$, which provides the final contradiction.

\section{Final remarks}

(1) If $\mathcal{F}$ is either a Fischer class containing $\mathcal{U}$ or the Fitting class product $\mathcal{N} \diamond \mathcal{H}$, $\mathcal{H}$ being a Fitting class containing $\mathcal{N}$, then $\mathcal{F}$ satisfies properties $(*)$ and $(* *)$ in Theorems 3.1 and 3.2 (see [9, Theorems 2 and 5]).

(2) If $\mathcal{F}$ is a Fitting class containing $\mathcal{U}$ and satisfying the property that $G / N \in \mathcal{F}$, whenever $G \in \mathcal{F}$ and $N \leqslant Z_{\mathcal{U}}(G)$ (in particular, if $\mathcal{F}$ is a Q-closed Fitting class), then $\mathcal{F}$ satisfies the property $(* *)$ in Theorem 3.1 (see [9, Theorem 3]).

(3) If $\mathcal{F}$ is an $\mathrm{R}_{0}$-closed Fitting class containing $\mathcal{U}$, then $\mathcal{F}$ satisfies the property $(* *)$ in Theorem 3.2 (see [9, Theorem 4]).

Acknowledgements. A.M.-P. and M.D.P.-R. were both supported by Proyecto BMF20001-1667-C03-03, Ministerio de Ciencia y Tecnología and FEDER, Spain.

\section{References}

1. A. Ballester-Bolinches and M. D. Pérez-Ramos, A question of R. Maier concerning formations, J. Alg. 182 (1996), 738-747.

2. A. Ballester-Bolinches, M. C. Pedraza-Aguilera and M. D. Pérez-Ramos, On finite products of totally permutable groups, Bull. Austral. Math. Soc. 53 (1996), 441-445.

3. A. Ballester-Bolinches, M. C. Pedraza-Aguilera and M. D. Pérez-Ramos, Finite groups which are products of pairwise totally permutable subgroups, Proc. Edinb. Math. Soc. 41 (1998), 567-572.

4. A. Ballester-Bolinches, M. C. Pedraza-Aguilera and M. D. Pérez-Ramos, Totally and mutually permutable products of finite groups, in Proc. of Groups St Andrews 1997, Bath, UK, vol. 1, pp. 65-68, London Mathematical Society Lecture Notes Series, vol. 260 (Cambridge University Press, 1999).

5. J. Beidleman and H. Heineken, Totally permutable torsion subgroups, J. Group Theory 2 (1999), 377-392.

6. A. CARocca, A note on the product of $\mathcal{F}$-subgroups in a finite group, Proc. Edinb. Math. Soc. 39 (1996), 37-42.

7. A. Carocca and R. Maike, Theorems of Kegel-Wielandt type, in Proc. of Groups St Andrews 1997, Bath, UK, vol. 1, pp. 195-201, London Mathematical Society Lecture Notes Series, vol. 260 (Cambridge University Press, 1999). 
8. K. Doerk And T. Hawkes, Finite soluble groups (Walter De Gruyter, Berlin, 1992).

9. P. Hauck, A. Martínez-Pastor and M. D. Pérez-Ramos, Fitting classes and products of totally permutable groups, J. Alg. 252 (2002), 114-126.

10. B. Huppert, Endliche Gruppen, vol. I (Springer, 1967).

11. R. Schmidt, Subgroup lattices of groups (Walter De Gruyter, Berlin, 1994). 